\title{
The Interpolating Boundary Element-Free Method for Unilateral Problems Arising in Variational Inequalities
}

\author{
Fen Li and Xiaolin Li \\ College of Mathematics Science, Chongqing Normal University, Chongqing 400047, China \\ Correspondence should be addressed to Xiaolin Li; lxlmath@163.com
}

Received 26 November 2013; Revised 30 December 2013; Accepted 31 December 2013; Published 12 February 2014

Academic Editor: Yumin Cheng

Copyright ( $2014 \mathrm{~F}$. Li and X. Li. This is an open access article distributed under the Creative Commons Attribution License, which permits unrestricted use, distribution, and reproduction in any medium, provided the original work is properly cited.

\begin{abstract}
The interpolating boundary element-free method (IBEFM) is developed in this paper for boundary-only analysis of unilateral problems which appear in variational inequalities. The IBEFM is a direct boundary only meshless method that combines an improved interpolating moving least-square scheme for constructing interpolation functions with boundary integral equations (BIEs) for representing governing equations. A projection operator is used to formulate the BIEs and then the formulae of the IBEFM are obtained for unilateral problems. The convergence of the developed meshless method is derived mathematically. The capability of the method is also illustrated and assessed through some numerical experiments.
\end{abstract}

\section{Introduction}

In the past two decades, considerable research effort in the field of computational science and engineering has been directed, both on theoretical and practical bases, into meshless (or meshfree) methods. Compared with the finite element method (FEM) and the boundary element method (BEM), meshless methods eliminate the difficulty of meshing and remeshing the considered computational structure via simply adding or deleting scattered nodes. The moving least square (MLS) [1] is an approximation scheme, which generates continuous functions from unorganized sampled point values. Since the numerical approximation of the MLS starts from scattered nodes instead of meshes, there have many meshless methods based on the MLS scheme. Among them are the diffuse element method, the element-free Galerkin (EFG) method, the $h-p$ meshless method, the meshless local Petrov-Galerkin method, and so on. These meshless methods followed the idea as the FEM, in which the problem domain is discretized.

Boundary integral equations (BIEs) are important and attractive computational tools as they can reduce the dimensionality of the considered problem by one. The MLS scheme has also been used in BIEs. Typical of them are the meshless local boundary integral equation (LBIE) method and the boundary node method (BNM) [2]. These BIEs-based meshless methods have emerged as promising numerical techniques in scientific computing. Nonetheless, since the MLS approximations lack the delta function property, boundary conditions in these meshless methods are difficult to be implemented. The technique used in the BNM doubles the number of both unknowns and system equations. Via combining a variational form of BIEs and the MLS scheme, another technique is developed in the symmetric Galerkin BNM to impose boundary conditions [3, 4]. However, in all these BIEs-based meshless methods, the basic unknown quantities are approximations of nodal variables, and therefore they are indirect methods. Recently, Cheng et al. [57] developed an improved MLS scheme that uses weighted orthogonal polynomials as basis functions. The improved MLS scheme has been introduced into BIEs to develop a direct meshless method, the boundary element-free method (BEFM) $[5,6,8,9]$. Because the improved MLS scheme still lacks the delta function property, boundary conditions in the BEFM are implemented with constraints.

To avoid the shortcomings of the MLS scheme, Liu and Gu proposed the point interpolation method (PIM) [10] and the radial PIM [11] to construct meshless shape functions. The PIMs have also been used in BIEs. Among them are the boundary PIMs [12-14], the hybrid boundary PIMs [15], 
and the hybrid radial BNM $[16,17]$. These meshless methods can exactly satisfy boundary conditions, since their shape functions possess delta function property.

To restore the delta function property of the MLS scheme, Lancaster and Salkauskas [1] further developed an interpolating moving least-square (IMLS) scheme that uses specific singular functions as weight functions. By revising the formula of the IMLS and combining it with BIEs, Ren et al. $[18,19]$ developed an interpolating boundary elementfree method (IBEFM) for 2D problems in potential theory and linear elasticity. In these improved methods, boundary conditions can be imposed with ease. A drawback of the IMLS scheme is that the involved weight function is singular at nodes. The singularity complicates the calculation of the inverse of the singular matrix and thus reduces the computing speed and efficiency.

To overcome the problems in both the MLS scheme and the IMLS scheme, Wang et al. [20, 21] proposed an improved interpolating moving least-square (IIMLS) scheme, an improved interpolating EFG method, and an IBEFM for 2D potential problems. In [22], Li discussed theoretically the properties of the IIMLS shape functions, studied the curve and surface fitting capability of the IIMLS scheme, and extended the IBEFM for 3D potential problems. Compared with the MLS scheme, the IIMLS scheme has the following features. Firstly, its shape function possesses the delta function property, so imposing boundary conditions in the IIMLS-based meshless method is much easier than that in the MLS-based meshless method. Secondly, the weight function used is nonsingular at any point; hence, any weight function used in the MLS scheme can also be used in the IIMLS scheme. Thirdly, the number of unknown coefficients in the trial function of the IIMLS scheme is less than that of the MLS scheme; thus, fewer nodes are required in the influence domain and a higher computational accuracy can be achieved in the IIMLS scheme.

In the theory of variational inequalities [23,24], a wide class of problems arising in industry, finance, economics, social, pure, and applied sciences give rise to the unilateral problem. The numerical solution of this problem has been frequently dominated by the FEM [25-28]. In the unilateral problem, the inequality boundary conditions are nonlinear and complementary ones which modeled threshold phenomena like contact problem, thermostatic device, or semipermeable membranes. As the inequality conditions lie on the unilateral contact boundary and this boundary is of prime interest, BIEs-based methods are ideally suited for the numerical solution. Some applications of the BEM for unilateral problems can be found in [29-36].

The present paper develops and employs the IBEFM for boundary-only analysis of unilateral problems. Using a projection operator, the unilateral problem is reacted as a sequence of well-posed bilateral problems. Then, the IBEFM is used iteratively for solving bilateral problems. As a result, only the boundary of the problem domain is discretized by properly scattered nodes. The convergence of the developed meshless method is also derived mathematically.

The following discussion begins with the brief description of the IIMLS scheme in Section 2. In Section 3, a detailed numerical implementation of the IBEFM for unilateral problems is presented. The convergence of this method is included in the next section. Then, numerical results are given in Section 5. Section 6 contains conclusions.

\section{The IIMLS Scheme for the IBEFM}

Consider a two-dimensional domain $\Omega$ bounded by its boundary $\Gamma$. In boundary-type meshless methods, only $\Gamma$ is represented using boundary nodes. The IIMLS scheme in this paper is independently generated on piecewise smooth segments $\Gamma_{i}\left(i=1,2, \ldots, N_{\Gamma}\right)$ which constitute the boundary naturally. Consequently, the problem of the discontinuity at corners can be avoided.

Let $\left\{p_{i}(s)\right\}_{i=0}^{m}$ be a given set of basis functions with $p_{0}(s) \equiv 1$, where $s$ is a curvilinear coordinate on $\Gamma$. Before generating shape functions with delta function property, we first construct the following basis functions:

$$
g_{i}(s, \bar{s})=p_{i}(\bar{s})-\sum_{j=1}^{n} q\left(s, s_{j}\right) p_{i}\left(s_{j}\right), \quad i=0,1, \ldots, m,
$$

where $s$ is the coordinate of an evaluation point on $\Gamma, s_{j}(j=$ $1,2, \ldots, n)$ are boundary nodes with influence domains that cover the point $s$, and the point $\bar{s}$ can be either the evaluation point $s$ or a nodal point $s_{j}$. The function $q\left(s, s_{j}\right)$ is taken such that

$$
q\left(s_{i}, s_{j}\right)=\delta_{i j}, \quad \sum_{j=1}^{n} q\left(s, s_{j}\right)=1, \quad \forall s \in \Gamma .
$$

Similar to the function used in $[20,21]$, this function is defined by

$$
\begin{array}{r}
q\left(s, s_{j}\right)=\frac{\xi\left(s, s_{j}\right)}{\sum_{k=1}^{n} \xi\left(s, s_{k}\right)}, \quad \xi\left(s, s_{j}\right)=\prod_{k=1, k \neq j}^{n} \frac{\left\|s-s_{k}\right\|^{2}}{\left\|s_{j}-s_{k}\right\|^{2}}, \\
1 \leq j \leq n,
\end{array}
$$

where $\|\cdot\|$ may denote any norm, but for reasons of differentiability it is natural to use the Euclidean norm.

For any $s \in \Gamma$, to obtain the IIMLS scheme for a given function $v(s)$, we define a new function $\widetilde{v}(s, \bar{s})$ as

$$
\widetilde{v}(s, \bar{s})=v(\bar{s})-\sum_{j=1}^{n} q\left(s, s_{j}\right) v\left(s_{j}\right)=v(\bar{s})-\mathbf{q}(s) \mathbf{v},
$$

where $\mathbf{q}(s)=\left[q\left(s, s_{1}\right), q\left(s, s_{2}\right), \ldots, q\left(s, s_{n}\right)\right]$ and $\mathbf{v}=\left[v\left(s_{1}\right)\right.$, $\left.v\left(s_{2}\right), \ldots, v\left(s_{n}\right)\right]^{\mathrm{T}}$.

In (1) letting $i=0$, then using (2), we have $g_{0}(s, \bar{s}) \equiv 0$. Thus, the local approximation $\widetilde{v}_{h}(s, \bar{s})$ of $\widetilde{v}(s, \bar{s})$ at $s$ can be defined as

$$
\widetilde{v}_{h}(s, \bar{s})=\sum_{j=1}^{m} g_{j}(s, \bar{s}) a_{j}(s)=\mathbf{g}(s, \bar{s}) \mathbf{a}(s),
$$

where $\mathbf{g}(s, \bar{s})=\left[g_{1}(s, \bar{s}), g_{2}(s, \bar{s}), \ldots, g_{m}(s, \bar{s})\right]$ and $\mathbf{a}(s)=$ $\left[a_{1}(s), a_{2}(s), \ldots, a_{m}(s)\right]^{\mathrm{T}}$. 
The coefficient vector $\mathbf{a}(s)$ is obtained by minimizing a weighted discrete $L^{2}$ norm defined as

$$
\begin{aligned}
J & =\sum_{i=1}^{n} w_{i}(s)\left[\widetilde{v}_{h}\left(s, s_{i}\right)-\widetilde{v}\left(s, s_{i}\right)\right]^{2} \\
& =\sum_{i=1}^{n} w_{i}(s)\left\{\mathbf{g}\left(s, s_{i}\right) \mathbf{a}(s)-\left[\mathbf{e}_{i}-\mathbf{q}(s)\right] \mathbf{v}\right\}^{2},
\end{aligned}
$$

where $w_{i}(s)$ are weight functions and $\mathbf{e}_{i}$ are $n$ unit row vectors with the $i$ th component being 1 .

The stationarity of $J$ with respect to $\mathbf{a}(s)$ yields

$$
\mathbf{a}(s)=\mathbf{A}^{-1}(s) \mathbf{B}(s) \mathbf{v},
$$

where

$$
\begin{gathered}
\mathbf{A}(s)=\sum_{i=1}^{n} w_{i}(s) \mathbf{g}^{\mathrm{T}}\left(s, s_{i}\right) \mathbf{g}\left(s, s_{i}\right), \\
\mathbf{B}(s)=\sum_{i=1}^{n} w_{i}(s) \mathbf{g}^{\mathrm{T}}\left(s, s_{i}\right)\left[\mathbf{e}_{i}-\mathbf{q}(s)\right] .
\end{gathered}
$$

Inserting (7) into (5) yields the local approximation

$$
\widetilde{v}_{h}(s, \bar{s})=\mathbf{g}(s, \bar{s}) \mathbf{A}^{-1}(s) \mathbf{B}(s) \mathbf{v} .
$$

Setting $\bar{s}=s$, then from (4) and (9), the global approximation of $v(s)$ is

$$
v_{h}(s)=\widetilde{v}_{h}(s, s)+\sum_{i=1}^{n} q\left(s, s_{i}\right) v\left(s_{i}\right)=\sum_{i=1}^{n} \Phi_{i}(s) v\left(s_{i}\right),
$$

where

$$
\Phi_{i}(s)=\sum_{j=1}^{m} g_{j}(s)\left[\mathbf{A}^{-1}(s) \mathbf{B}(s)\right]_{j i}+q\left(s, s_{i}\right), \quad i=1,2, \ldots, n,
$$

are the IIMLS shape functions, and $g_{j}(s)$ are defined by (1) as

$$
g_{j}(s)=p_{j}(s)-\sum_{k=1}^{n} q\left(s, s_{k}\right) p_{j}\left(s_{k}\right), \quad j=1,2, \ldots, m .
$$

To generate the IIMLS shape functions, the $m \times m$ moment matrix $\mathbf{A}(s)$ needs to be invertible. As in the MLS scheme $[3,4]$, a necessary condition for the invertibility of $\mathbf{A}(s)$ is that there are at least $m+1$ nodes covered in the influence domain of the point $s$. Besides, (5) indicates that the number of the unknown coefficients $a_{j}(s)$ in the IIMLS scheme is fewer than that in the MLS scheme. As a result, fewer nodes can be chosen in the meshless method formed with the IIMLS scheme compared to that formed with the MLS scheme. Moreover, compared with the IMLS scheme developed in $[1,18,19]$, any weight function used in the MLS scheme can also be used in the IIMLS scheme.

We list below some propositions of the IIMLS shape function $\Phi_{i}(s)$. The detailed mathematical proof can be found in $[22]$.
Proposition 1. The influence domain of the node $s_{i}$ is the compact support of $\Phi_{i}(s), i=1,2, \ldots, n$.

Proposition 2. $\Phi_{i}(s)$ is of delta function properties as

$$
\Phi_{i}\left(\mathbf{x}_{j}\right)=\delta_{i j}=\left\{\begin{array}{ll}
1, & i=j, \\
0, & i \neq j,
\end{array} \quad i, j=1,2, \ldots, n .\right.
$$

Proposition 3. $\Phi_{i}(s)$ is of reproducing properties as

$$
\begin{array}{r}
\sum_{i=1}^{n} \Phi_{i}(s)=1, \quad \sum_{i=1}^{n} \Phi_{i}(s) p_{l}\left(s_{i}\right)=p_{l}(s), \\
l=1,2, \ldots, m,
\end{array}
$$

where $p_{l}(s)$ is the given basis function.

\section{The IBEFM for Unilateral Problems}

We consider the unilateral problem in the bounded domain $\Omega$ with a smooth boundary $\Gamma$. Let $\Gamma_{D}, \Gamma_{N}$ and $\Gamma_{C}$ be mutually disjoint parts of $\Gamma$ such that $\Gamma=\Gamma_{D} \cup \Gamma_{N} \cup \Gamma_{C}$, and $\Gamma_{C} \neq \emptyset$. Given $\phi \in H^{1 / 2}\left(\Gamma_{D} \cup \Gamma_{C}\right)$ and $\varphi \in H^{-1 / 2}\left(\Gamma_{N} \cup \Gamma_{C}\right)$, we want to find the solution $u \in H^{1}(\Omega)$ satisfying

$$
\begin{gathered}
\Delta u=0, \quad \text { in } \Omega, \\
u=\phi, \quad \text { on } \Gamma_{D}, \\
q=\varphi, \quad \text { on } \Gamma_{N}, \\
u \geq \phi, \quad q \geq \varphi, \quad(u-\phi)(q-\varphi)=0, \quad \text { on } \Gamma_{C},
\end{gathered}
$$

where $q=\partial u / \partial \mathbf{n}$ is the normal derivative of $u$ and $\mathbf{n}$ is the unit outward normal to $\Gamma$.

We introduce the Sobolev space $\mathscr{H}(\Omega)$ and the convex cone $\mathscr{K}(\Omega)$ of admissible functions which satisfy the noninterpenetration on the unilateral contact boundary $\Gamma_{C}$ :

$$
\begin{aligned}
& \mathscr{H}(\Omega)=\left\{v \in H^{1}(\Omega): v=\phi, \text { on } \Gamma_{D}\right\}, \\
& \mathscr{K}(\Omega)=\left\{v \in \mathscr{H}(\Omega): v \geq \phi, \text { on } \Gamma_{C}\right\} .
\end{aligned}
$$

Then the unilateral problem (15)-(16) is equivalent to the following variational inequality over $\Omega$ [27]:

$$
\begin{gathered}
\text { find } u \in \mathscr{K}(\Omega) \text { such that } \\
\int_{\Omega} \nabla v \nabla(v-u) \mathrm{d} \Omega \geq \int_{\Gamma_{N} \cup \Gamma_{C}} \varphi(v-u) \mathrm{d} \Gamma, \quad \forall v \in \mathscr{K}(\Omega) .
\end{gathered}
$$

It was proved in [30] that this variational inequality admits a unique solution if and only if $\Gamma_{D} \neq \emptyset$ or $\int_{\Gamma_{N} \cup \Gamma_{C}} \varphi d \Gamma<0$. Besides, let

$$
\begin{gathered}
\mathscr{H}(\Gamma)=\left\{v \in H^{1 / 2}(\Gamma): v=\phi, \text { on } \Gamma_{D}\right\}, \\
\mathscr{K}(\Gamma)=\left\{v \in \mathscr{H}(\Gamma): v \geq \phi, \text { on } \Gamma_{C}\right\},
\end{gathered}
$$


then from Green's formula it follows that variational inequality (18) may equivalently be formulated as the following boundary variational inequality on $\Gamma$ :

find $u \in \mathscr{K}(\Gamma)$ such that

$$
\int_{\Gamma} q(v-u) \mathrm{d} \Gamma \geq \int_{\Gamma_{N} \cup \Gamma_{C}} \varphi(v-u) \mathrm{d} \Gamma, \quad \forall v \in \mathscr{K}(\Gamma) .
$$

Obviously, the unilateral problem (15)-(16) and variational inequalities (18) and (20) are equivalent.

In the unilateral problem (15)-(16), the inequality boundary conditions (16) are nonlinear and represent the contact with an unilateral boundary obstacle given by the function $\phi$. In such problems, we need to determine the parts of $\Gamma_{C}$ on which conditions $u=\phi$ and $q \geq \varphi$ apply and its remaining parts on which conditions $u \geq \phi$ and $q=\varphi$ apply.

The purpose of this paper is to develop a meshless method for the numerical solution of the unilateral problem (15)-(16), but difficulties arise in the implementation of the unilateral contact conditions (16). To tackle this issue, we define the projection operator $\mathscr{P}: \mathbb{R} \rightarrow \mathbb{R}^{+} \cup\{0\}$ by $[23,24,32,37]$

$$
\mathscr{P} v=\max (0, v), \quad v \in \mathbb{R} .
$$

It can be easily verified that the inequality boundary conditions (16) are equivalent to the following condition:

$$
q-\varphi=\mathscr{P}[(q-\varphi)-\alpha(u-\phi)], \quad \text { on } \Gamma_{C},
$$

where $\alpha$ is an arbitrary but fixed positive constant. Then, we define an implicit iterative scheme on $\Gamma_{C}$ as

$$
\begin{array}{r}
q^{(k+1)}=\varphi+\mathscr{P}\left[\left(q^{(k)}-\varphi\right)-\alpha\left(u^{(k+1)}-\phi\right)\right], \\
k=0,1,2, \ldots
\end{array}
$$

As in [32, 37], the unilateral problem is therefore converted into a sequence of well-posed bilateral problems with straightforward boundary conditions. And then, the IBEFM is used to solve these problems. For this purpose, we check the boundary conditions on $\Gamma_{C}$. If on some parts of $\Gamma_{C}$, denoted as $\Gamma_{C N}$, inequality

$$
\left(q^{(k)}-\varphi\right)-\alpha\left(u^{(k)}-\phi\right)<0
$$

is true, then the boundary condition is imposed as

$$
q^{(k+1)}=\varphi, \quad \text { on } \Gamma_{C N},
$$

for the next iteration. On the remaining parts of $\Gamma_{C}$, denoted as $\Gamma_{C R}$, the boundary condition is imposed as

$$
q^{(k+1)}=q^{(k)}-\alpha\left(u^{(k+1)}-\phi\right), \quad \text { on } \Gamma_{C R} .
$$

Equations (15), (25), and (26) compose the following wellposed bilateral problem:

$$
\begin{gathered}
\Delta u^{(k+1)}=0, \quad \text { in } \Omega, \\
u^{(k+1)}=\phi, \quad \text { on } \Gamma_{D}, \\
q^{(k+1)}=\varphi, \quad \text { on } \Gamma_{N} \cup \Gamma_{C N}, \\
q^{(k+1)}=q^{(k)}-\alpha\left(u^{(k+1)}-\phi\right), \quad \text { on } \Gamma_{C R} .
\end{gathered}
$$

In the following, problem (27) is solved by the IBEFM to obtain $u^{(k+1)}$ and $q^{(k+1)}$ on $\Gamma_{C}$.

The BIE for the solution of (27) is [38]

$$
\begin{aligned}
C(\mathbf{y}) u^{(k+1)}(\mathbf{y})= & \int_{\Gamma} u^{*}(\mathbf{x}, \mathbf{y}) q^{(k+1)}(\mathbf{x}) \mathrm{d} \Gamma \\
& -\int_{\Gamma} q^{*}(\mathbf{x}, \mathbf{y}) u^{(k+1)}(\mathbf{x}) \mathrm{d} \Gamma, \quad \mathbf{y} \in \Gamma,
\end{aligned}
$$

where $\mathbf{x}=\left(x_{1}, x_{2}\right)$ is the field point, $\mathbf{y}=\left(y_{1}, y_{2}\right)$ is the source point, $u^{*}(\mathbf{x}, \mathbf{y})=-(1 / 2 \pi) \ln |\mathbf{x}-\mathbf{y}|$ and $q^{*}(\mathbf{x}, \mathbf{y})=$ $\partial u^{*}(\mathbf{x}, \mathbf{y}) / \partial \mathbf{n}(\mathbf{y})$ are fundamental solutions, and $C(\mathbf{y})$ is the function of the internal angle that the boundary $\Gamma$ makes at the point $y$.

From the expression of the approximation function (10), we let

$$
\begin{aligned}
& u^{(k+1)}(\mathbf{x})=\sum_{j=1}^{N} \Phi_{j}(\mathbf{x}) u_{j}^{(k+1)}, \\
& q^{(k+1)}(\mathbf{x})=\sum_{j=1}^{N} \Phi_{j}(\mathbf{x}) q_{j}^{(k+1)},
\end{aligned}
$$

where $N$ is the number of nodes on the boundary $\Gamma, u_{j}^{(k+1)}=$ $u^{(k+1)}\left(\mathbf{x}_{j}\right), q_{j}^{(k+1)}=q^{(k+1)}\left(\mathbf{x}_{j}\right)$, and $\Phi_{j}(\mathbf{x})$ is the contributions from the boundary node $\mathbf{x}_{j}$ to the evaluation point $\mathbf{x}$. In the influence domain of the $j$ th node, $\Phi_{j}(\mathbf{x})$ is the meshless shape function given by (11) and is not equal to zero. Otherwise, $\Phi_{j}(\mathbf{x})$ is equal to zero.

Substituting (29) into (28) for all boundary nodes $\left\{\mathbf{y}_{i}\right\}_{i=1}^{N}$ yields

$$
\begin{aligned}
C\left(\mathbf{y}_{i}\right) u^{(k+1)}\left(\mathbf{y}_{i}\right)= & \sum_{j=1}^{N} q_{j}^{(k+1)} \int_{\Gamma} u^{*}\left(\mathbf{x}, \mathbf{y}_{i}\right) \Phi_{j}(\mathbf{x}) \mathrm{d} \Gamma \\
& -\sum_{j=1}^{N} u_{j}^{(k+1)} \int_{\Gamma} q^{*}\left(\mathbf{x}, \mathbf{y}_{i}\right) \Phi_{j}(\mathbf{x}) \mathrm{d} \Gamma .
\end{aligned}
$$

Thus, we have the following linear algebraic equations:

$$
\mathbf{H U}=\mathbf{G Q} \text {, }
$$

where $\mathbf{U}=\left(u_{1}^{(k+1)}, u_{2}^{(k+1)}, \ldots, u_{N}^{(k+1)}\right)^{\mathrm{T}}, \mathbf{Q}=\left(q_{1}^{(k+1)}, q_{2}^{(k+1)}\right.$, $\left.\ldots, q_{N}^{(k+1)}\right)^{\mathrm{T}}$, and $\mathbf{H}$ and $\mathbf{G}$ are the corresponding coefficient matrices.

To obtain the unknowns at boundary nodes, boundary conditions needed to be imposed. For the convenience of discussion, we assume that the first $N_{d}$ boundary nodes $\left\{\mathbf{y}_{i}\right\}_{i=1}^{N_{d}}$ belong to $\Gamma_{D}$, the next $N_{n}$ boundary nodes $\left\{\mathbf{y}_{i}\right\}_{i=N_{d}+1}^{N_{d}+N_{n}}$ belong to $\Gamma_{N} \cup \Gamma_{C N}$, and the rest $N_{r}=N-N_{d}-N_{n}$ boundary nodes $\left\{\mathbf{y}_{i}\right\}_{i=N_{d}+N_{n}+1}^{N}$ belong to $\Gamma_{C R}$. Then by applying 
the boundary conditions given on $\Gamma_{D}$ and $\Gamma_{N} \cup \Gamma_{C N}$, we can rewrite (31) as the following block form:

$$
\begin{aligned}
{\left[\begin{array}{lll}
\mathbf{H}_{11} & \mathbf{H}_{12} & \mathbf{H}_{13} \\
\mathbf{H}_{21} & \mathbf{H}_{22} & \mathbf{H}_{23} \\
\mathbf{H}_{31} & \mathbf{H}_{32} & \mathbf{H}_{33}
\end{array}\right]\left[\begin{array}{l}
\overline{\mathbf{U}}_{1} \\
\mathbf{U}_{2} \\
\mathbf{U}_{3}
\end{array}\right] } \\
=\left[\begin{array}{lll}
\mathbf{G}_{11} & \mathbf{G}_{12} & \mathbf{G}_{13} \\
\mathbf{G}_{21} & \mathbf{G}_{22} & \mathbf{G}_{23} \\
\mathbf{G}_{31} & \mathbf{G}_{32} & \mathbf{G}_{33}
\end{array}\right]\left[\begin{array}{l}
\mathbf{Q}_{1} \\
\overline{\mathbf{Q}}_{2} \\
\mathbf{Q}_{3}
\end{array}\right],
\end{aligned}
$$

where $\overline{\mathbf{U}}_{1}=\left(u_{1}^{(k+1)}, \ldots, u_{N_{d}}^{(k+1)}\right)^{\mathrm{T}}$ and $\overline{\mathbf{Q}}_{2}=\left(q_{N_{d}+1}^{(k+1)}\right.$, $\left.\ldots, q_{N_{d}+N_{n}}^{(k+1)}\right)^{\mathrm{T}}$ are given, and $\mathbf{Q}_{1}=\left(q_{1}^{(k+1)}, \ldots, q_{N_{d}}^{(k+1)}\right)^{\mathrm{T}}, \mathbf{U}_{2}=$ $\left(u_{N_{d}+1}^{(k+1)}, \ldots, u_{N_{d}+N_{n}}^{(k+1)}\right)^{\mathrm{T}}, \mathbf{U}_{3}=\left(u_{N_{d}+N_{n}+1}^{(k+1)}, \ldots, u_{N}^{(k+1)}\right)^{\mathrm{T}}$, and $\mathbf{Q}_{3}=$ $\left(q_{N_{d}+N_{n}+1}^{(k+1)}, \ldots, q_{N}^{(k+1)}\right)^{\mathrm{T}}$ are unknown. Besides, collocating the Robin boundary conditions (26) at nodes $\left\{\mathbf{y}_{i}\right\}_{i=N_{d}+N_{n}+1}^{N} \subset \Gamma_{C R}$ yields

$$
q_{i}^{(k+1)}=q_{i}^{(k)}-\alpha\left(u_{i}^{(k+1)}-\phi_{i}\right), \quad i=N_{d}+N_{n}+1, \ldots, N,
$$

where $\phi_{i}=\phi\left(\mathbf{y}_{i}\right)$. It can be expressed as

$$
\mathbf{Q}_{3}=\overline{\mathbf{F}}-\alpha \mathbf{U}_{3},
$$

where $\overline{\mathbf{F}}=\left(f_{N_{d}+N_{n}+1}, f_{N_{d}+N_{n}+2}, \ldots, f_{N}\right)^{\mathrm{T}}$ with $f_{i}=q_{i}^{(k)}+\alpha \phi_{i}$. Substituting (34) into (32) and taking all unknowns to the left side lead to the following $N \times N$ linear algebraic equations:

$$
\begin{gathered}
{\left[\begin{array}{lll}
-\mathbf{G}_{11} & \mathbf{H}_{12} & \mathbf{H}_{13}+\alpha \mathbf{G}_{13} \\
-\mathbf{G}_{21} & \mathbf{H}_{22} & \mathbf{H}_{23}+\alpha \mathbf{G}_{23} \\
-\mathbf{G}_{31} & \mathbf{H}_{32} & \mathbf{H}_{33}+\alpha \mathbf{G}_{33}
\end{array}\right]\left[\begin{array}{l}
\mathbf{Q}_{1} \\
\mathbf{U}_{2} \\
\mathbf{U}_{3}
\end{array}\right]} \\
=\left[\begin{array}{lll}
-\mathbf{H}_{11} & \mathbf{G}_{12} & \mathbf{G}_{13} \\
-\mathbf{H}_{21} & \mathbf{G}_{22} & \mathbf{G}_{23} \\
-\mathbf{H}_{31} & \mathbf{G}_{32} & \mathbf{G}_{33}
\end{array}\right]\left[\begin{array}{l}
\overline{\mathbf{U}}_{1} \\
\overline{\mathbf{Q}}_{2} \\
\overline{\mathbf{F}}
\end{array}\right] .
\end{gathered}
$$

Solving (35), we can obtain unknowns $\mathbf{Q}_{1}, \mathbf{U}_{2}$, and $\mathbf{U}_{3}$. Then inserting $\mathbf{U}_{3}$ into (34), the unknown $\mathbf{Q}_{3}$ can be obtained directly.

The IBEFM flow chart for the unilateral problem (15)-(16) can be concluded as follows.

Algorithm 4. Choose a tolerance $\varepsilon>0$ and set $k=0$.

(1) Select $N$ nodes on boundary $\Gamma$ and compute shape functions.

(2) Assume initially that the boundary condition on the unilateral boundary $\Gamma_{C}$ is

$$
q^{(0)}=\varphi, \quad \text { on } \Gamma_{C},
$$

and then (15) and (36) compose a well-posed problem and are solved together by the IBEFM to obtain $u^{(0)}$ on $\Gamma_{C}$.

(3) Determine $\Gamma_{C N}$ and $\Gamma_{C R}$ by checking the boundary conditions on $\Gamma_{C}$ and then form the boundary value problem (27).
(4) Obtain the linear algebra equations (35).

(5) Solve (35) and (34) to obtain $u_{i}^{(k+1)}$ and $q_{i}^{(k+1)}$ for all nodes $\mathbf{y}_{i} \in \Gamma_{C}$.

(6) Compute the relative error

$$
e(u)=\sqrt{\frac{\sum_{i}\left(u_{i}^{(k+1)}-u_{i}^{(k)}\right)^{2}}{\sum_{i}\left(u_{i}^{(k+1)}\right)^{2}}} .
$$

(7) Stop the algorithm if $e(u) \leq \varepsilon$. Otherwise, update $k$ to $k+1$ and go to (3)

In this paper, the allowable tolerance used is $\varepsilon=10^{-6}$. Stop condition of $e(u) \leq \varepsilon$ determines the number of iterations.

\section{The Convergence of the Developed Meshless Algorithm}

The mathematical proof of convergence of some mesh-based numerical algorithms has been established [23, 24, 28, 30, $32,35]$. In this section, a convergence study of the proposed meshless algorithm is carried out.

Lemma 5. Let $\left(u^{*}, q^{*}\right)$ be the exact solution of the unilateral problem (15)-(16), and let $\left\{\left(u^{(k)}, q^{(k)}\right)\right\}$ be the sequence generated by Algorithm 4, and then

$$
\begin{gathered}
\left\langle u^{(k+1)}-\phi, q^{(k)}-q^{*}\right\rangle_{\Gamma_{C}} \geq\left\langle u^{(k+1)}-\phi, q^{(k)}-q^{(k+1)}\right\rangle_{\Gamma_{C}}, \\
\left\|q^{(k+1)}-q^{*}\right\|_{\Gamma_{C}}^{2} \leq \\
-\left\|q^{(k)}-\alpha\left(u^{(k+1)}-\phi\right)-q^{*}\right\|_{\Gamma_{C}}^{2} \\
-\left\|q^{(k+1)}-q^{(k)}+\alpha\left(u^{(k+1)}-\phi\right)\right\|_{\Gamma_{C}}^{2},
\end{gathered}
$$

where the duality $\langle\cdot, \cdot\rangle_{\Gamma_{C}}$ is defined for functions $w$ and $v$ as $\langle w, v\rangle_{\Gamma_{C}}=\int_{\Gamma_{C}} w v d \Gamma$, and the norm $\|\cdot\|_{\Gamma_{C}}$ is defined as $\|v\|_{\Gamma_{C}}=$ $\sqrt{\langle v, v\rangle_{\Gamma_{C}}}$.

Proof. Using Green's formula leads to

$$
\begin{gathered}
\left\langle u^{(k+1)}-\phi, q^{(k+1)}-q^{*}\right\rangle_{\Gamma_{C}}-\left\langle u^{*}-\phi, q^{(k+1)}-q^{*}\right\rangle_{\Gamma_{C}} \\
=\left\langle u^{(k+1)}-u^{*}, q^{(k+1)}-q^{*}\right\rangle_{\Gamma_{C}} \\
=\left\|\nabla\left(u^{(k+1)}-u^{*}\right)\right\|_{L^{2}(\Omega)}^{2} \geq 0 .
\end{gathered}
$$

Thus applying (16) and (23) yields

$$
\begin{aligned}
\left\langle u^{(k+1)}\right. & \left.-\phi, q^{(k+1)}-q^{*}\right\rangle_{\Gamma_{C}} \\
& \geq\left\langle u^{*}-\phi, q^{(k+1)}-q^{*}\right\rangle_{\Gamma_{C}} \\
& =\left\langle u^{*}-\phi, q^{(k+1)}-\varphi\right\rangle_{\Gamma_{C}}-\left\langle u^{*}-\phi, q^{*}-\varphi\right\rangle_{\Gamma_{C}} \\
& =\left\langle u^{*}-\phi, q^{(k+1)}-\varphi\right\rangle_{\Gamma_{C}} \geq 0 .
\end{aligned}
$$


Therefore,

$$
\begin{aligned}
\left\langle u^{(k+1)}-\phi, q^{(k)}-q^{*}\right\rangle_{\Gamma_{C}} & =\left\langle u^{(k+1)}-\phi, q^{(k)}-q^{(k+1)}\right\rangle_{\Gamma_{C}} \\
& +\left\langle u^{(k+1)}-\phi, q^{(k+1)}-q^{*}\right\rangle_{\Gamma_{C}} \\
\geq & \left\langle u^{(k+1)}-\phi, q^{(k)}-q^{(k+1)}\right\rangle_{\Gamma_{C}} .
\end{aligned}
$$

On the other hand, if $\left(q^{(k)}-\varphi\right)-\alpha\left(u^{(k+1)}-\phi\right)<0$ on $\Gamma_{C}$, then (23) indicates $q^{(k+1)}=\varphi$; thus

$$
q^{(k+1)}-q^{(k)}+\alpha\left(u^{(k+1)}-\phi\right)>0,
$$

and using $q^{*}-\varphi \geq 0$ yields

$$
q^{(k+1)}-q^{*} \leq 0 .
$$

Otherwise, if $\left(q^{(k)}-\varphi\right)-\alpha\left(u^{(k+1)}-\phi\right) \geq 0$ on $\Gamma_{C}$, then applying (23) leads to

$$
\begin{aligned}
& q^{(k+1)}-q^{(k)}+\alpha\left(u^{(k+1)}-\phi\right) \\
& \quad=q^{(k+1)}-\varphi-\mathscr{P}\left[\left(q^{(k)}-\varphi\right)-\alpha\left(u^{(k+1)}-\phi\right)\right]=0 .
\end{aligned}
$$

Briefly, we have

$$
\left\langle q^{(k+1)}-q^{(k)}+\alpha\left(u^{(k+1)}-\phi\right), q^{(k+1)}-q^{*}\right\rangle_{\Gamma_{\mathrm{C}}} \leq 0 .
$$

Consequently,

$$
\begin{aligned}
\left\|q^{(k+1)}-q^{*}\right\|_{\Gamma_{C}}^{2} & \\
= & \| q^{(k+1)}-\left(q^{(k)}-\alpha\left(u^{(k+1)}-\phi\right)\right) \\
& +\left(q^{(k)}-\alpha\left(u^{(k+1)}-\phi\right)\right)-q^{*} \|_{\Gamma_{C}}^{2} \\
= & \left\|q^{(k)}-q^{*}-\alpha\left(u^{(k+1)}-\phi\right)\right\|_{\Gamma_{C}}^{2} \\
& -\left\|q^{(k+1)}-q^{(k)}+\alpha\left(u^{(k+1)}-\phi\right)\right\|_{\Gamma_{C}}^{2} \\
& +2\left\langle q^{(k+1)}-q^{(k)}+\alpha\left(u^{(k+1)}-\phi\right), q^{(k+1)}-q^{*}\right\rangle_{\Gamma_{C}} \\
\leq & \left\|q^{(k)}-q^{*}-\alpha\left(u^{(k+1)}-\phi\right)\right\|_{\Gamma_{C}}^{2} \\
& -\left\|q^{(k+1)}-q^{(k)}+\alpha\left(u^{(k+1)}-\phi\right)\right\|_{\Gamma_{C}}^{2},
\end{aligned}
$$

which completes the proof.

Theorem 6. Under the conditions of Lemma 5 ,

$$
\lim _{k \rightarrow \infty}\left(u^{(k)}, q^{(k)}\right)=\left(u^{*}, q^{*}\right) .
$$

Proof. According to (39), we obtain

$$
\begin{aligned}
\left\|q^{(k+1)}-q^{*}\right\|_{\Gamma_{C}}^{2} & \\
\leq \| q^{(k)}- & q^{*}\left\|_{\Gamma_{C}}^{2}-\right\| q^{(k+1)}-q^{(k)} \|_{\Gamma_{C}}^{2} \\
-2 \alpha & \left(\left\langle u^{(k+1)}-\phi, q^{(k)}-q^{*}\right\rangle_{\Gamma_{C}}\right. \\
& \left.+\left\langle u^{(k+1)}-\phi, q^{(k+1)}-q^{(k)}\right\rangle_{\Gamma_{C}}\right) .
\end{aligned}
$$

Then invoking (38) yields

$$
\left\|q^{(k+1)}-q^{*}\right\|_{\Gamma_{C}}^{2} \leq\left\|q^{(k)}-q^{*}\right\|_{\Gamma_{C}}^{2}-\left\|q^{(k+1)}-q^{(k)}\right\|_{\Gamma_{C}}^{2},
$$

which indicates

$$
\left\|q^{(k+1)}-q^{*}\right\|_{\Gamma_{\mathrm{C}}} \leq\left\|q^{(k)}-q^{*}\right\|_{\Gamma_{\mathrm{C}}} .
$$

Besides, the sequence $\left\{q^{(k)}\right\}$ is bounded and

$$
\begin{aligned}
& \sum_{k=0}^{\infty}\left\|q^{(k+1)}-q^{(k)}\right\|_{\Gamma_{C}}^{2} \\
& \quad \leq \sum_{k=0}^{\infty}\left(\left\|q^{(k)}-q^{*}\right\|_{\Gamma_{C}}^{2}-\left\|q^{(k+1)}-q^{*}\right\|_{\Gamma_{C}}^{2}\right) \\
& \leq\left\|q^{(0)}-q^{*}\right\|_{\Gamma_{C}}^{2}
\end{aligned}
$$

Thus, $\left\{q^{(k)}\right\}$ is a Cauchy sequence.

Let $\left(u^{*}, q^{*}\right)$ be the cluster point of the sequence $\left\{\left(u^{(k)}, q^{(k)}\right)\right\}$ and the subsequence $\left\{\left(u^{\left(k_{1}\right)}, q^{\left(k_{1}\right)}\right)\right\}$ of $\left\{\left(u^{(k)}, q^{(k)}\right)\right\}$ converges to $\left(u^{*}, q^{*}\right)$, and then $\left(u^{*}, q^{*}\right)$ satisfies (22). Since (16) and (22) are equivalent, $\left(u^{*}, q^{*}\right)$ is a solution of the unilateral problem (15)-(16).

In order to prove that $\left(u^{*}, q^{*}\right)$ is the unique cluster point of $\left\{\left(u^{(k)}, q^{(k)}\right)\right\}$, we now assume that $\left(u^{* *}, q^{* *}\right)$ is another cluster point and let

$$
\delta=\left\|q^{* *}-q^{*}\right\|_{\Gamma_{C}} .
$$

Because $\left(u^{*}, q^{*}\right)$ is the cluster point, there exists a positive integer $\bar{k}$ such that

$$
\left\|q^{(\bar{k})}-q^{*}\right\|_{\Gamma_{\mathrm{C}}} \leq \frac{\delta}{2} .
$$

Then, for any $k>\bar{k}$, applying (51) leads to

$$
\left\|q^{(k)}-q^{*}\right\|_{\Gamma_{C}} \leq \frac{\delta}{2} .
$$

So,

$$
\left\|q^{(k)}-q^{* *}\right\|_{\Gamma_{\mathrm{C}}} \geq\left\|q^{* *}-q^{*}\right\|_{\Gamma_{\mathrm{C}}}-\left\|q^{(k)}-q^{*}\right\|_{\Gamma_{\mathrm{C}}} \geq \frac{\delta}{2},
$$

which implies

$$
\lim _{k \rightarrow \infty} q^{(k)} \neq q^{* *}
$$


Consequently, the sequence $\left\{\left(u^{(k)}, q^{(k)}\right)\right\}$ has only one cluster point, and thus we conclude that $\left\{\left(u^{(k)}, q^{(k)}\right)\right\}$ converges to the exact solution of the unilateral problem (15)-(16).

Theorem 6 indicates that the developed meshless method generates a sequence of strongly convergent approximations.

\section{Numerical Experiments}

5.1. Spann Problem. In [30], Spann constructed a test problem in an annular domain with an explicitly known solution. The exact solution is

$$
\begin{array}{r}
u\left(x_{1}, x_{2}\right)=\operatorname{Im}\left(\sqrt{A+B} \operatorname{sign} x_{1}+i \sqrt{A-B} \operatorname{sign} x_{2}\right)^{3}, \\
\text { in } \Omega=\left\{a<\sqrt{x_{1}^{2}+x_{2}^{2}}<b\right\},
\end{array}
$$

where

$$
\begin{gathered}
A=\frac{1}{2} \sqrt{\left(\frac{x_{1}^{2}-x_{2}^{2}}{x_{1}^{2}+x_{2}^{2}}\right)^{2}+\frac{1}{4}\left(\frac{x_{1}^{2}+x_{2}^{2}-a^{2}}{a^{2}\left(x_{1}^{2}+x_{2}^{2}\right)}\right)^{2}}, \\
B=\frac{\left(x_{1}^{2}-x_{2}^{2}\right)\left(x_{1}^{2}+x_{2}^{2}+a^{2}\right)}{4 a^{2}\left(x_{1}^{2}+x_{2}^{2}\right)^{2}} .
\end{gathered}
$$

In this problem, Dirichlet boundary conditions are imposed on the outer circle corresponding to the exact solution, and unilateral boundary conditions are imposed on the inner circle as

$$
\begin{array}{r}
u \geq \phi, \quad q \geq \varphi, \quad(u-\phi)(q-\varphi)=0, \\
\text { on } \Gamma_{C}=\left\{\sqrt{x_{1}^{2}+x_{2}^{2}}=a\right\},
\end{array}
$$

where $\phi\left(x_{1}, x_{2}\right)=\min \left(0, u\left(x_{1}, x_{2}\right)\right)$ and

$$
\begin{aligned}
& \varphi\left(x_{1}, x_{2}\right) \\
& = \begin{cases}-\frac{6}{a}, & x_{2}>0, \\
-\frac{6}{a^{5}}\left(x_{1}^{2}-x_{2}^{2}\right)^{2}, & x_{2} \leq 0, x_{2} \geq-\left|x_{1}\right|, \\
0, & x_{2}<-\left|x_{1}\right| .\end{cases}
\end{aligned}
$$

As in [30], the parameters are chosen as $a=0.1$ and $b=0.25$ in computation. Our discretization includes 64 boundary nodes on each circle. The exact solution and the numerical solution for $u$ on the unilateral contact boundary $\Gamma_{C}$ are presented in Figure 1. The results for $q$ are presented in Figure 2. In these figures, we use the parameterization $t \rightarrow$ $(a \cos 2 \pi t,-a \sin 2 \pi t)$. From these figures, we can see that the numerical solutions are in excellent agreement with the exact solutions.

To study the convergence, the $L^{2}$ relative error norms of $u$ on $\Gamma_{C}$ obtained by the IBEFM are plotted in Figure 3 in log$\log$ scale. In this figure, the results of the Galerkin boundary element method (GBEM) [30], the boundary element-linear

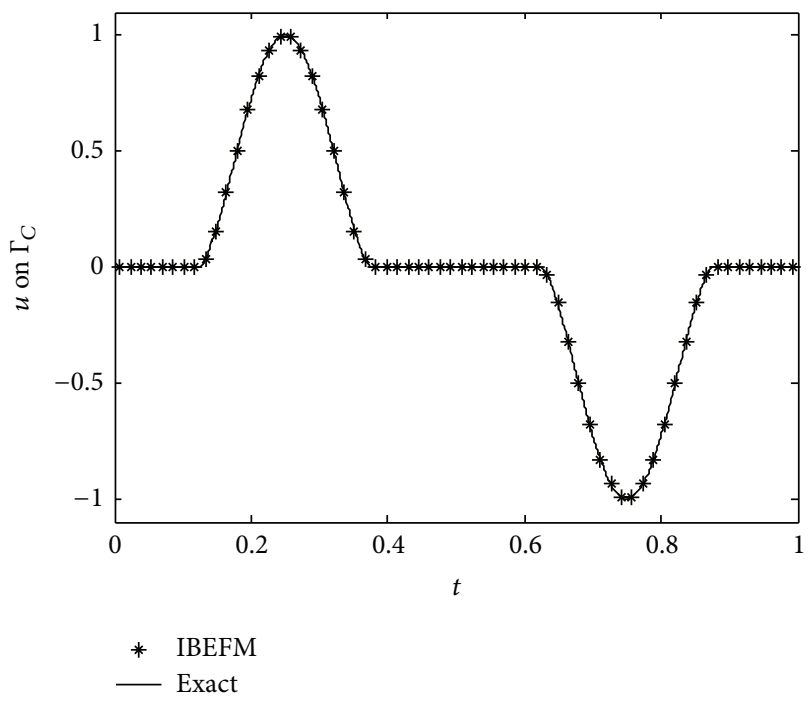

Figure 1: Exact and numerical solutions for $u$ on $\Gamma_{C}$.

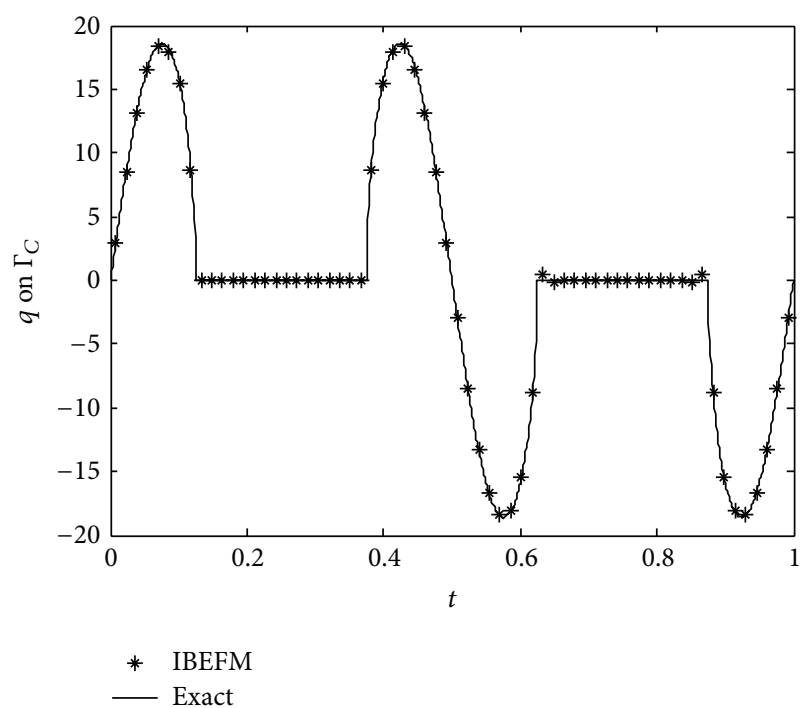

Figure 2: Exact and numerical solutions for $q$ on $\Gamma_{C}$.

complementary method (BE-LCM) [34], and the boundary element-projection iterative method (BE-PIM) [35] are also given for comparison. It is shown that the error of the IBEFM is the least. Besides, the convergence rate obtained from the GBEM, the BE-LCM, the BE-PIM, and the IBEFM is found to be $1.11,1.01,1.84$, and 2.00 , respectively.

The corresponding number of iterations is shown in Table 1, from which we can see that the number of iterations of the IBEFM and the BE-PIM is little influenced by the number of boundary nodes and is less than that of the GBEM and the BE-LCM. Besides, Table 2 gives the corresponding CPU times. We can find that CPU times of the IBEFM are less than both the BE-LCM and the BE-PIM. As a result, the computing speed and efficiency of the IBEFM are higher than those of the GBEM, the BE-LCM and the BE-PIM. 
TABLE 1: Number of iterations for Spann problem.

\begin{tabular}{|c|c|c|c|c|c|c|}
\hline & \multicolumn{6}{|c|}{$N$} \\
\hline & 32 & 64 & 128 & 256 & 512 & 1024 \\
\hline GBEM & 24 & 28 & 36 & 48 & & \\
\hline BE-LCM & 56 & 91 & 141 & 256 & 263 & 319 \\
\hline BE-PIM & 4 & 4 & 5 & 6 & 7 & 8 \\
\hline IBEFM & 5 & 6 & 6 & 7 & 8 & 8 \\
\hline
\end{tabular}

TABLE 2: CPU times (in seconds) for Spann problem.

\begin{tabular}{lcccccc}
\hline & & & $N$ & & \\
& 32 & 64 & 128 & 256 & 1024 \\
\hline BE-LCM & 0.047 & 0.125 & 0.453 & 2.407 & 16.953 & 125.907 \\
BE-PIM & 0.078 & 0.141 & 0.438 & 1.782 & 8.141 & 40.188 \\
IBEFM & 0.062 & 0.094 & 0.203 & 0.609 & 2.484 & 11.218 \\
\hline
\end{tabular}

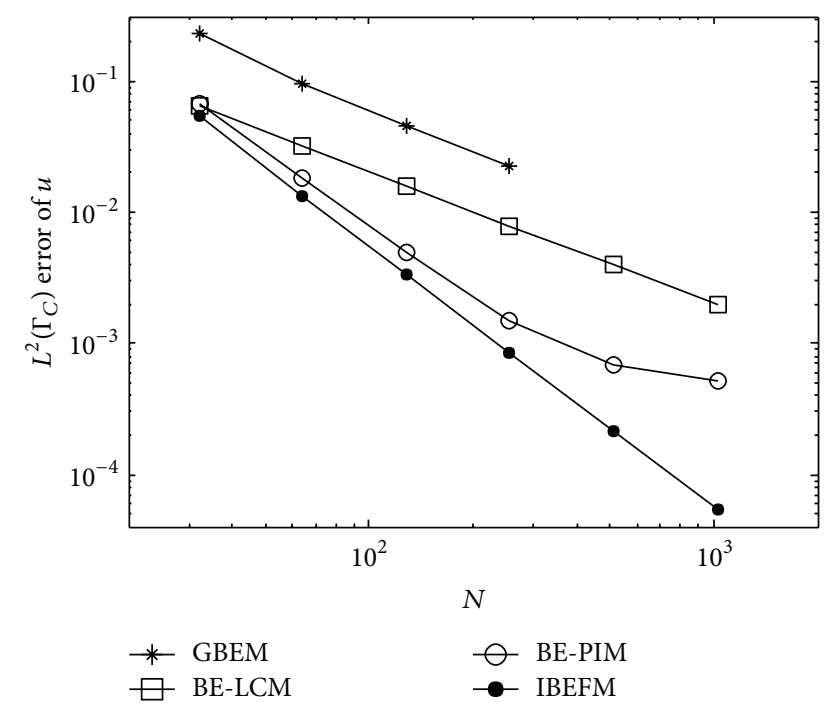

FIgUre 3: Convergence of the BEM and the IBEFM for Spann problem.

5.2. Groundwater Flow Problem. The problem solved here is a groundwater flow problem related to percolation in gently sloping beaches [25]. This problem can be formulated as a free boundary problem for the pressure. In the saturated region, the pressure $u$ is a harmonic function satisfying

$$
\begin{gathered}
\Delta u=0, \quad \text { in } \Omega=(0,1) \times(0,1), \\
u=0, \quad \text { on } \Gamma_{D}=\left\{\left(x_{1}, x_{2}\right): x_{1}=0,0 \leq x_{2} \leq 1\right\}, \\
u=G(1), \quad \text { on } \Gamma_{D}=\left\{\left(x_{1}, x_{2}\right): x_{1}=1,0 \leq x_{2} \leq 1\right\}, \\
\frac{\partial u}{\partial \mathbf{n}}=0, \quad \text { on } \Gamma_{N}=\left\{\left(x_{1}, x_{2}\right): 0 \leq x_{1} \leq 1, x_{2}=0\right\}, \\
\frac{\partial u}{\partial \mathbf{n}}=0, \quad u<G\left(x_{1}\right), \\
\text { on } \Gamma_{C}=\left\{\left(x_{1}, x_{2}\right): 0 \leq x_{1} \leq 1, x_{2}=1\right\},
\end{gathered}
$$

or

$$
u=G\left(x_{1}\right), \quad \frac{\partial u}{\partial \mathbf{n}}<0, \quad \text { on } \Gamma_{C}
$$

where $G\left(x_{1}\right)$ is a known function depicting the surface profile. Clearly, the numerical solution of this problem relies on $G\left(x_{1}\right)$.

We consider two cases of surface profiles given in $[25,30]$

$$
\begin{gathered}
G_{1}\left(x_{1}\right)=\left(\frac{1}{2}-x_{1}\right)\left(1-x_{1}\right)-x_{1}, \\
G_{2}\left(x_{1}\right)=\sin \left(12 x_{1}\right)-2 .
\end{gathered}
$$

The numerical results are plotted in Figure 4. In this analysis, 32 boundary nodes are used to discretize per side of the domain. The first profile yields only one separation point, while the second profile yields four separation points. There are no exact solutions available in the literature to verify the developed meshless method for such a complex problem. However, we can observe that the results are in good agreement with those given in $[25,30]$.

In order to investigate the convergence of the IBEFM, the problem is solved by choosing different numbers of boundary nodes. Since the exact solution of the problem is not known, the error is estimated by substituting the exact solutions with some selected approximate values which can be computed by more boundary nodes. In this work, we obtain these approximate values by using 4096 boundary nodes. Figure 5 plots the log-log plot of the $L^{2}$ relative error norms of $u$ on $\Gamma_{C}$ with respect to the number of nodes. It can be seen that, as the number of nodes increases, the error becomes smaller. Besides, the experimental convergence rate for $G_{1}\left(x_{1}\right)$ and $G_{2}\left(x_{1}\right)$ is 2.02 and 2.15 , respectively. Therefore, the experimental convergence rate is very high and approximate to 2. 


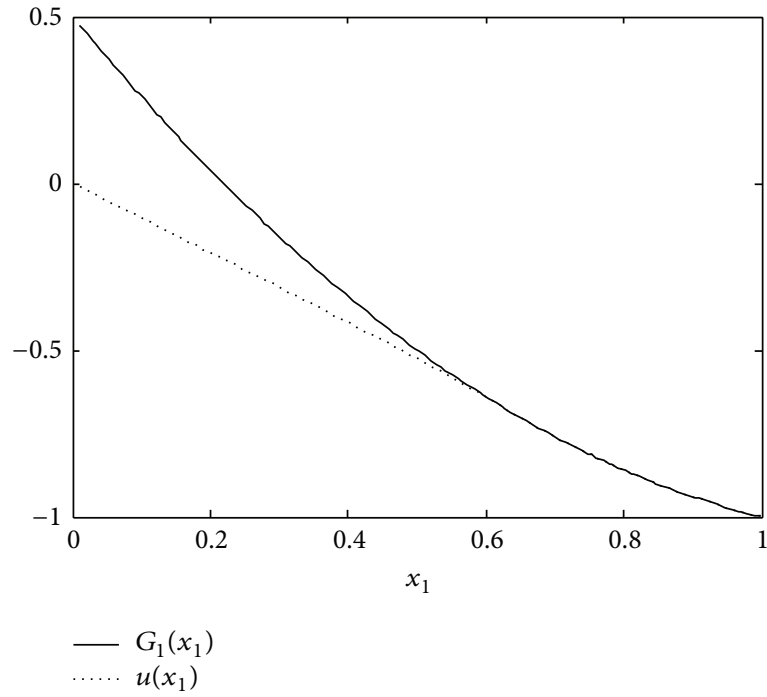

(a)

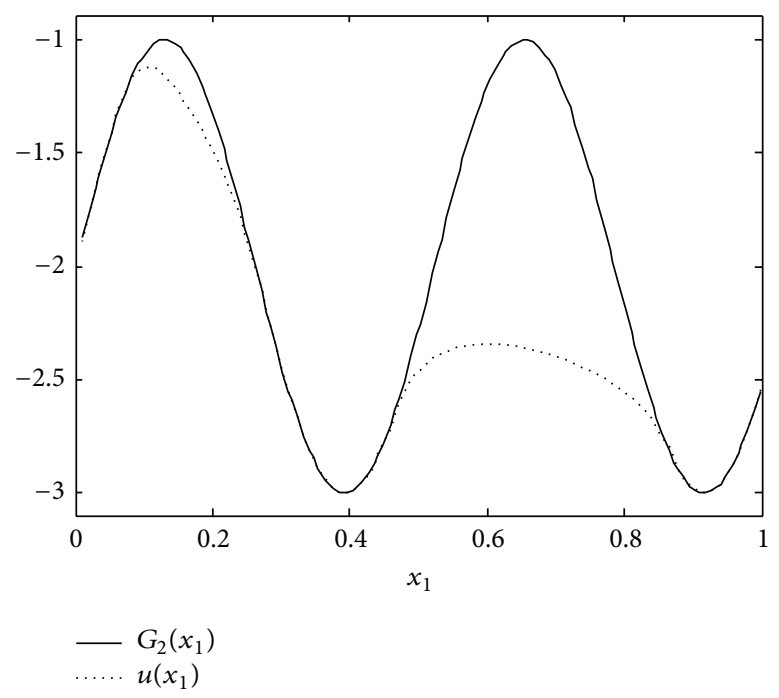

(b)

FIGURE 4: Approximate solution of the groundwater flow problem with (a) $G_{1}\left(x_{1}\right)$ and (b) $G_{2}\left(x_{1}\right)$.

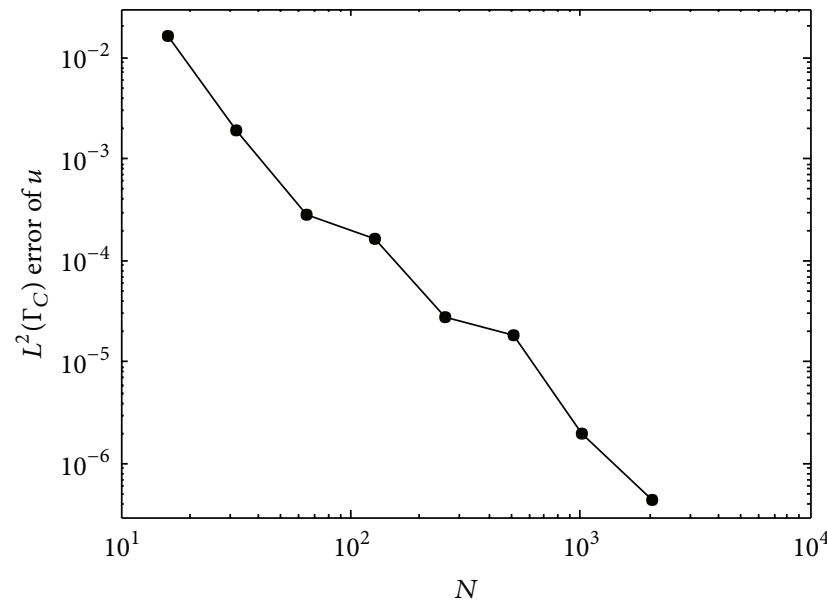

(a)

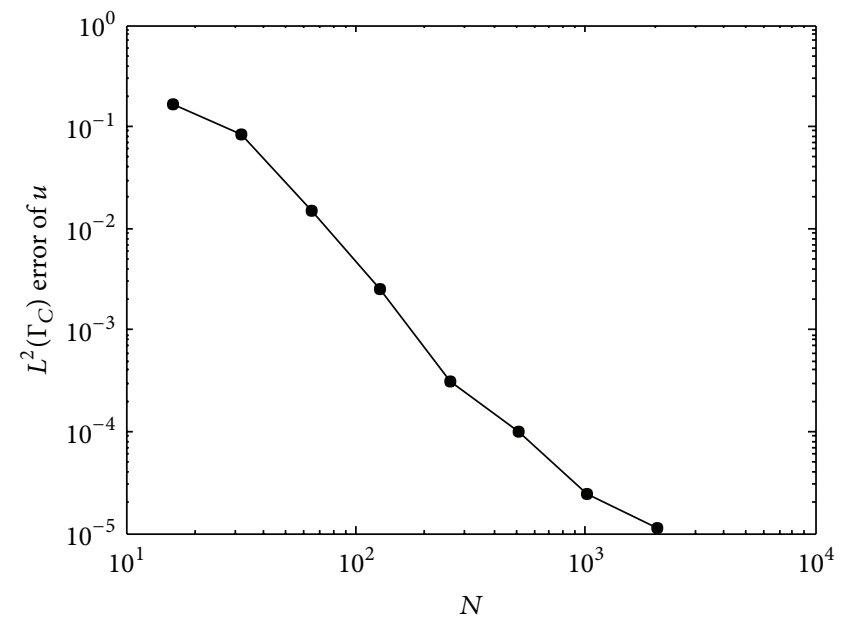

(b)

Figure 5: Convergence of the IBEFM for the groundwater flow problem with (a) $G_{1}\left(x_{1}\right)$ and (b) $G_{2}\left(x_{1}\right)$.

\section{Conclusion}

A novel boundary-type meshless method, the IBEFM, is developed in this paper for solving unilateral problems arising from variational inequalities. The IBEFM is based on BIEs discretized with the IIMLS scheme that only uses a group of arbitrarily distributed boundary nodes. In this numerical algorithm, the nonlinear inequality boundary conditions are incorporated into the iterative scheme naturally, and boundary conditions can be imposed with ease. Convergence proof of this meshless method has been provided mathematically. The proof shows that this method generates a sequence of strongly convergent approximations.

Some numerical examples have been given to validate the capacity of the method. For all examples, a convergent solution has been gained. The numerical results have verified the accuracy, convergence and effectiveness of the IBEFM. The experimental convergence rate is very high and approximate to 2. The errors and CPU times were compared to those obtained by the BEMs. These comparisons show that the IBEFM provides monotonic convergence and high accuracy results with low computational expenses.

Initial numerical results from the IBEFM, applied to 2D unilateral problems, are encouraging. The numerical experiments indicate that the developed meshless method has not only the higher convergence rate and computational precision but also the less CPU times than the BEMs presented in $[34,35]$. Besides, due to its considerable flexibility in mesh generation and relative ease in implementation, this method has tremendous potential for efficiently solving $3 \mathrm{D}$ 
problems and problems with complex domains to take the full advantages of the meshless concept. Nevertheless, more research work is required.

\section{Conflict of Interests}

The authors declare that there is no conflict of interests regarding the publication of this paper.

\section{Acknowledgments}

This work was supported by the National Natural Science Foundation of China under Grant no. 11101454, the Educational Commission Foundation of Chongqing of China under Grant no. KJ130626, the Natural Science Foundation Project of CQ CSTC under Grant no. cstc2013jcyjA30001, and the Program of Chongqing Innovation Team Project in University under Grant no. KJTD201308.

\section{References}

[1] P. Lancaster and K. Salkauskas, "Surfaces generated by moving least squares methods," Mathematics of Computation, vol. 37, no. 155, pp. 141-158, 1981.

[2] Y. Xie Mukherjee and S. Mukherjee, "The boundary node method for potential problems," International Journal for Numerical Methods in Engineering, vol. 40, no. 5, pp. 797-815, 1997.

[3] X. Li and J. Zhu, "A Galerkin boundary node method and its convergence analysis," Journal of Computational and Applied Mathematics, vol. 230, no. 1, pp. 314-328, 2009.

[4] X. Li, "Meshless Galerkin algorithms for boundary integral equations with moving least square approximations," Applied Numerical Mathematics, vol. 61, no. 12, pp. 1237-1256, 2011.

[5] Y. Cheng and M. Chen, "A boundary element-free method for linear elasticity," Acta Mechanica Sinica, vol. 35, no. 2, pp. 181186,2003

[6] K. M. Liew, Y. Cheng, and S. Kitipornchai, "Boundary elementfree method (BEFM) and its application to two-dimensional elasticity problems," International Journal for Numerical Methods in Engineering, vol. 65, no. 8, pp. 1310-1332, 2006.

[7] Z. Zhang, J. Wang, Y. Cheng, and K. M. Liew, "The improved element-free Galerkin method for three-dimensional tr heat conduction problems," Science China Physics, Mechanics \& Astronomy, vol. 56, no. 8, pp. 1568-1580, 2013.

[8] Y. Cheng and M. Peng, "Boundary element-free method for elastodynamics," Science in China, Series G, vol. 48, no. 6, pp. 641-657, 2005.

[9] M. Peng and Y. Cheng, "A boundary element-free method (BEFM) for two-dimensional potential problems," Engineering Analysis with Boundary Elements, vol. 33, no. 1, pp. 77-82, 2009.

[10] G. R. Liu and Y. T. Gu, "A point interpolation method for two-dimensional solids," International Journal for Numerical Methods in Engineering, vol. 50, no. 4, pp. 937-951, 2001.

[11] J. G. Wang and G. R. Liu, "A point interpolation meshless method based on radial basis functions," International Journal for Numerical Methods in Engineering, vol. 54, no. 11, pp. 16231648, 2002.
[12] Y. T. Gu and G. R. Liu, "A boundary point interpolation method for stress analysis of solids," Computational Mechanics, vol. 28, no. 1, pp. 47-54, 2002.

[13] Y. T. Gu and G. R. Liu, "A boundary radial point interpolation method (BRPIM) for 2-D structural analyses," Structural Engineering and Mechanics, vol. 15, no. 5, pp. 535-550, 2003.

[14] G. R. Liu and Y. T. Gu, "Boundary meshfree methods based on the boundary point interpolation methods," Engineering Analysis with Boundary Elements, vol. 28, no. 5, pp. 475-487, 2004.

[15] Y. T. Gu and G. R. Liu, "Hybrid boundary point interpolation methods and their coupling with the element free Galerkin method," Engineering Analysis with Boundary Elements, vol. 27, no. 9, pp. 905-917, 2003.

[16] X. Li, J. Zhu, and S. Zhang, "A hybrid radial boundary node method based on radial basis point interpolation," Engineering Analysis with Boundary Elements, vol. 33, no. 11, pp. 1273-1283, 2009.

[17] X. Li, "Numerical solution of solid mechanics problems using a boundary-only and truly meshless method," Mathematical Problems in Engineering, vol. 2012, Article ID 298903, 16 pages, 2012.

[18] H.-P. Ren, Y.-M. Cheng, and W. Zhang, "An improved boundary element-free method (IBEFM) for two-dimensional potential problems," Chinese Physics B, vol. 18, no. 10, pp. 4065-4073, 2009.

[19] H. Ren, Y. Cheng, and W. Zhang, "An interpolating boundary element-free method (IBEFM) for elasticity problems," Science China: Physics, Mechanics \& Astronomy, vol. 53, no. 4, pp. 758766, 2010.

[20] J. Wang, F. Sun, and Y. Cheng, "An improved interpolating element-free Galerkin method with nonsingular weight function for two-dimensional potential problems," Chinese Physics $B$, vol. 21, no. 9, Article ID 090204, 2012.

[21] J. Wang, J. Wang, F. Sun, and Y. Cheng, "An interpolating boundary element-free method with nonsingular weight function for two-dimensional potential problems," International Journal of Computational Methods, vol. 10, no. 6, Article ID 135004, 23 pages, 2013.

[22] X. Li, "An interpolating boundary element-free method for three-dimensional potential problems," Applied Mathematical Modelling. In press.

[23] M. A. Noor, "Projection iterative methods for extended general variational inequalities," Journal of Applied Mathematics and Computing, vol. 32, no. 1, pp. 83-95, 2010.

[24] J. Y. Han, N. H. Xiu, and H. D. Qi, Nonlinear Complementarity Theory and Algorithm, Shanghai Science and Technology Press, Shanghai, China, 2006.

[25] J. M. Aitchison, C. M. Elliott, and J. R. Ockendon, "Percolation in gently sloping beaches," IMA Journal of Applied Mathematics, vol. 30, no. 3, pp. 269-287, 1983.

[26] J. M. Aitchison, A. A. Lacey, and M. Shillor, "A model for an electropaint process," IMA Journal of Applied Mathematics, vol. 33, no. 1, pp. 17-31, 1984.

[27] R. Glowinski, Numerical Methods for Nonlinear Variational Problems, Springer Series in Computational Physics, Springer, New York, NY, USA, 1984.

[28] P. Coorevits, P. Hild, K. Lhalouani, and T. Sassi, "Mixed finite element methods for unilateral problems: convergence analysis and numerical studies," Mathematics of Computation, vol. 71, no. 237, pp. 1-25, 2002. 
[29] A. Karageorghis, "Numerical solution of a shallow dam problem by a boundary element method," Computer Methods in Applied Mechanics and Engineering, vol. 61, no. 3, pp. 265-276, 1987.

[30] W. Spann, "On the boundary element method for the Signorini problem of the Laplacian," Numerische Mathematik, vol. 65, no. 3, pp. 337-356, 1993.

[31] J.-T. Chen, C.-C. Hsiao, Y.-P. Chiu, and Y.-T. Lee, "Study of free-surface seepage problems using hypersingular equations," Communications in Numerical Methods in Engineering, vol. 23, no. 8, pp. 755-769, 2007.

[32] J. M. Aitchison and M. W. Poole, "A numerical algorithm for the solution of Signorini problems," Journal of Computational and Applied Mathematics, vol. 94, no. 1, pp. 55-67, 1998.

[33] C. Polizzotto, "Variational boundary-integral-equation approach to unilateral contact problems in elasticity," Computational Mechanics, vol. 13, no. 1-2, pp. 100-115, 1993.

[34] S. Zhang and J. Zhu, "The boundary element-linear complementarity method for the Signorini problem," Engineering Analysis with Boundary Elements, vol. 36, no. 2, pp. 112-117, 2012.

[35] S. Zhang and J. Zhu, "A projection iterative algorithm boundary element method for the Signorini problem," Engineering Analysis with Boundary Elements, vol. 37, no. 1, pp. 176-181, 2013.

[36] T. Panzeca, M. Salerno, S. Terravecchia, and L. Zito, "The symmetric boundary element method for unilateral contact problems," Computer Methods in Applied Mechanics and Engineering, vol. 197, no. 33-40, pp. 2667-2679, 2008.

[37] K. Ito and K. Kunisch, "Semi-smooth Newton methods for the Signorini problem," Applications of Mathematics, vol. 53, no. 5, pp. 455-468, 2008.

[38] J. Zhu and Z. Yuan, Boundary Element Analysis, Science Press, Beijing, China, 2009. 


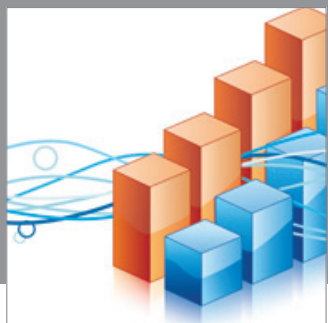

Advances in

Operations Research

mansans

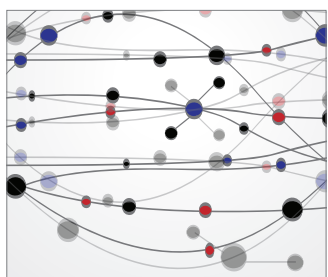

The Scientific World Journal
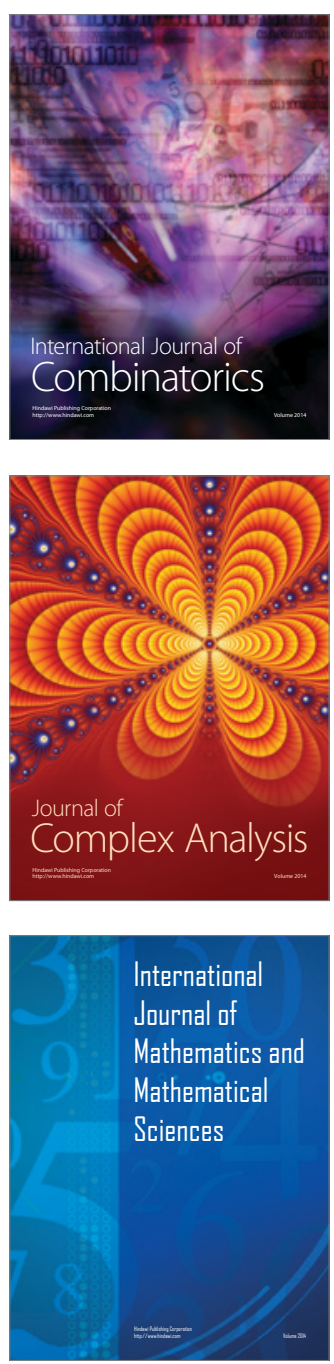
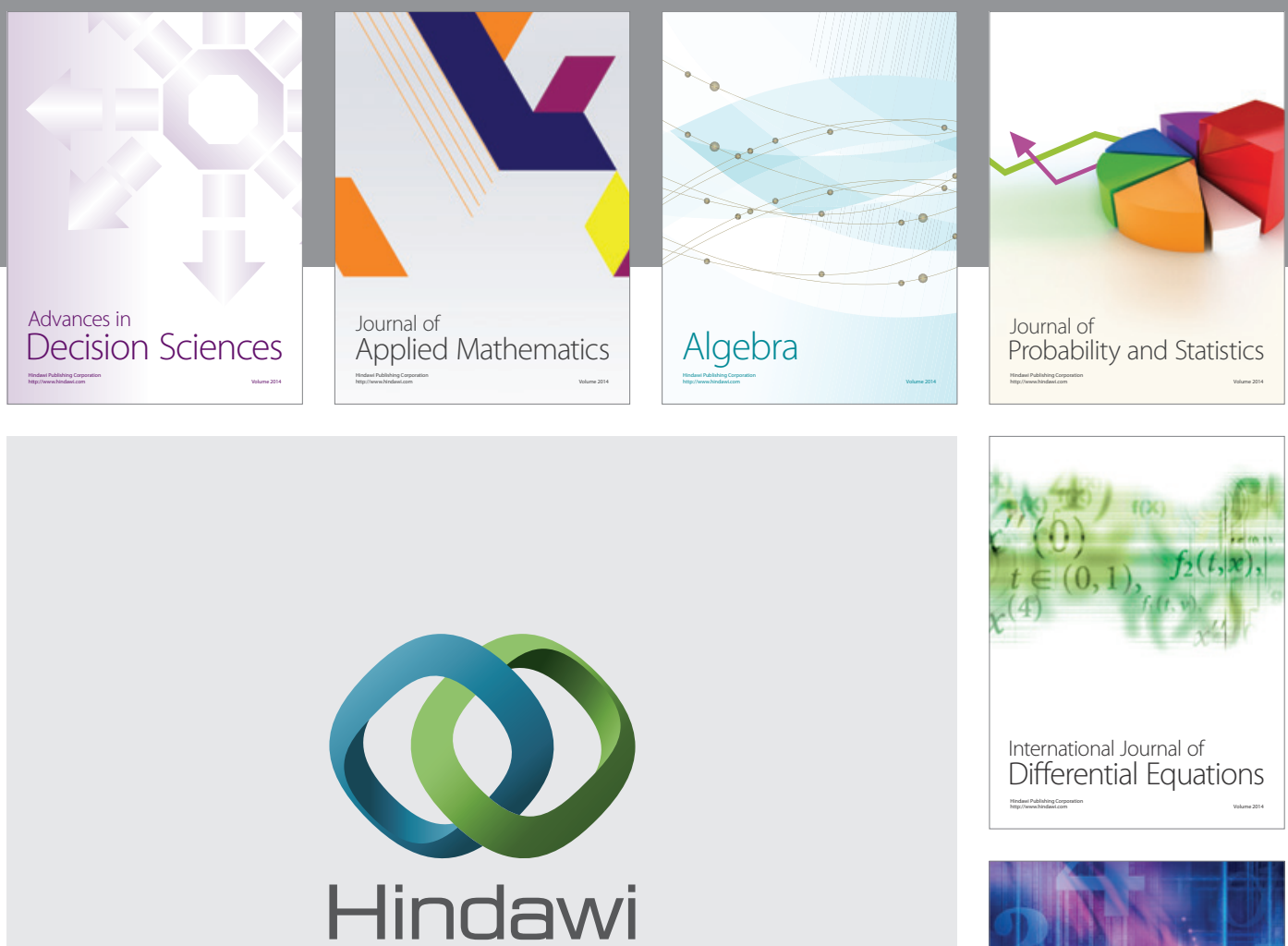

Submit your manuscripts at http://www.hindawi.com
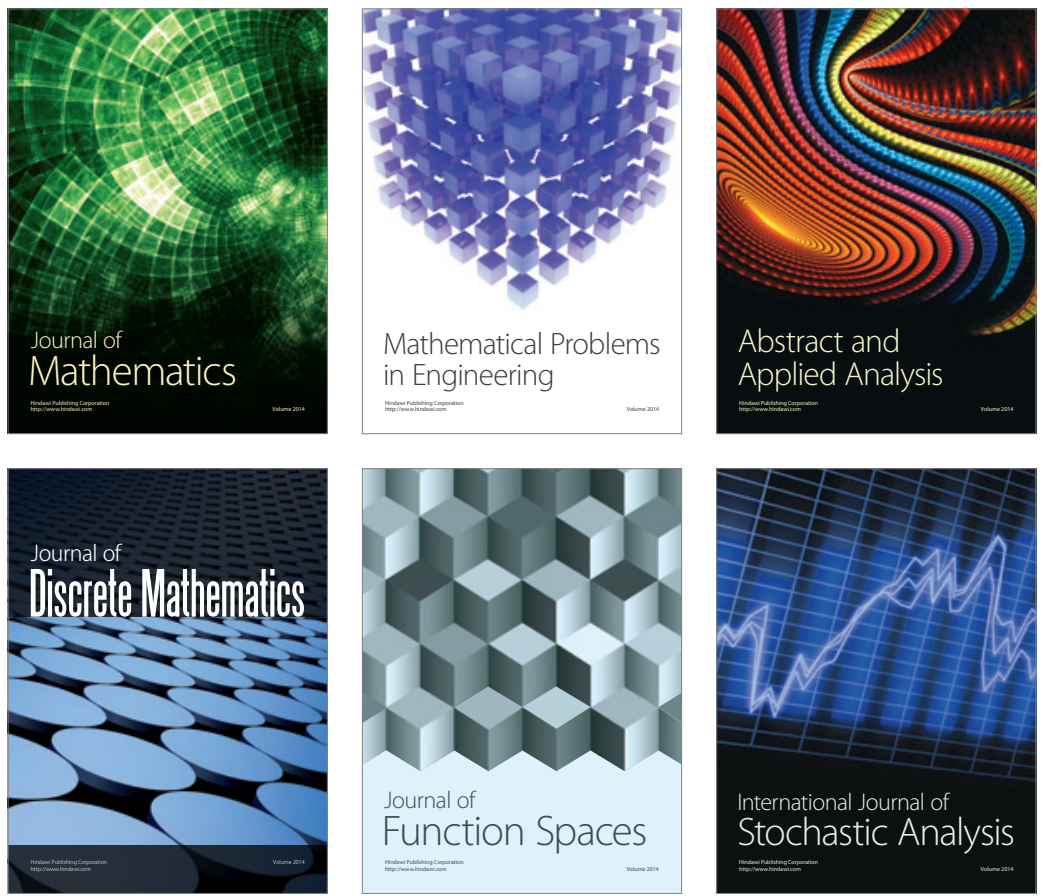

Journal of

Function Spaces

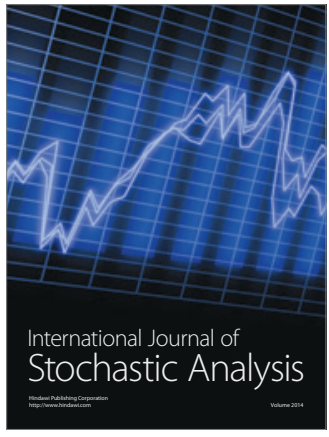

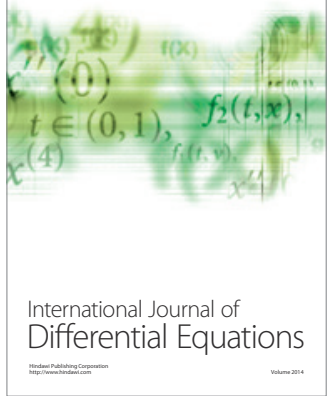
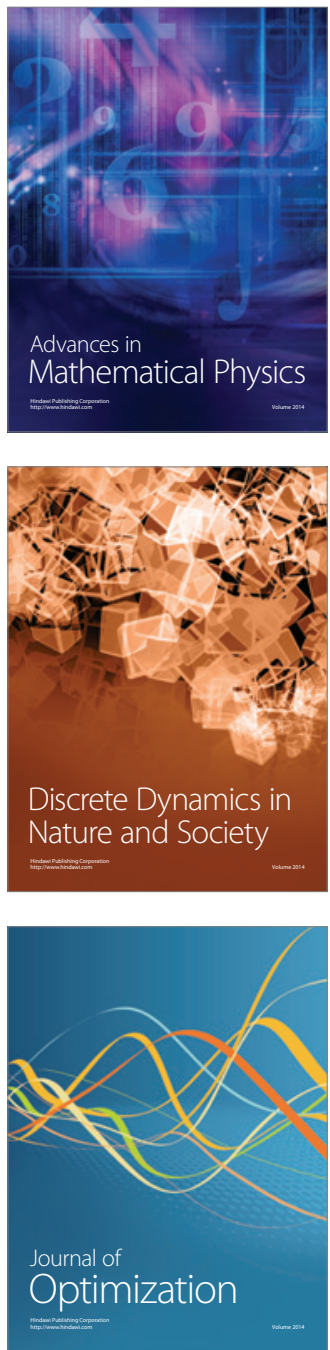